\title{
Point Defects and $p$-Type Doping in ScN from First Principles
}

\author{
Yu Kumagai, ${ }^{1,2, *}$ Naoki Tsunoda, ${ }^{3}$ and Fumiyasu Oba ${ }^{3,1,4}$ \\ ${ }^{1}$ Materials Research Center for Element Strategy, Tokyo Institute of Technology, \\ Yokohama 226-8503, Japan \\ ${ }^{2}$ PRESTO, Japan Science and Technology Agency, Tokyo 113-8656, Japan \\ ${ }^{3}$ Laboratory for Materials and Structures, Institute of Innovative Research, \\ Tokyo Institute of Technology, Yokohama 226-8503, Japan \\ ${ }^{4}$ Center for Materials Research by Information Integration, National Institute for Materials Science, \\ Tsukuba 305-0047, Japan
}

(Received 8 October 2017; revised manuscript received 24 January 2018; published 22 March 2018)

\begin{abstract}
Scandium nitride $(\mathrm{ScN})$ has been intensively researched as a prototype of rocksalt nitrides and a potential counterpart of the wurtzite group IIIa nitrides. It also holds great promise for applications in various fields, including optoelectronics, thermoelectrics, spintronics, and piezoelectrics. We theoretically investigate the bulk properties, band-edge positions, chemical stability, and point defects, i.e., native defects, unintentionally doped impurities, and p-type dopants of $\mathrm{ScN}$ using the Heyd-Scuseria-Ernzerhof hybrid functional. We find several fascinating behaviors: (i) a high level for the valence-band maximum, (ii) the lowest formation energy among binary nitrides, (iii) high formation energies of native point defects, (iv) low formation energies of donor-type impurities, and (v) a p-type conversion by $\mathrm{Mg}$ doping. Furthermore, we uncover the origins of the Burstein-Moss shift commonly observed in ScN. Our work sheds light on a fundamental understanding of $\mathrm{ScN}$ in regard to its technological applications.
\end{abstract}

DOI: 10.1103/PhysRevApplied.9.034019

\section{INTRODUCTION}

Group IIIa nitrides, i.e., AlN, GaN, and InN, have been massively investigated for various applications, including the best-known commercialization of light-emitting diodes [1-4]. Other nitrides have also been intensively studied recently, thanks to the progress of bulk crystal and film growth techniques $[5,6]$. One class of nitrides consists of $R \mathrm{~N}$ nitrides, where $R$ indicates a rare-earth element. Among them, $\mathrm{ScN}$ has been most researched as a prototype of rocksalt (RS) nitrides and a counterpart of the wurtzite (WZ) IIIa nitrides. In addition, $\mathrm{ScN}$ holds great promise for applications by virtue of its high melting point [7] and mechanical hardness [8]. Furthermore, it has attracted significant interest in recent years for its applications in thermoelectrics (the record is $3.5 \times 10^{-3} \mathrm{~W} / \mathrm{mK}^{2}$ ) $[9,10]$, spintronics [11], and piezoelectrics [12-15], and for tuning the optical gaps of the IIIa nitrides by alloying [16-18].

$\mathrm{ScN}$ is known to show an indirect-type band structure, and its optical gap is increased by increasing the

yuuukuma@gmail.com

Published by the American Physical Society under the terms of the Creative Commons Attribution 4.0 International license. Further distribution of this work must maintain attribution to the author(s) and the published article's title, journal citation, and DOI. concentration of carrier electrons that fills the lower part of the conduction band. Such an increase of the optical gap is known as the Burstein-Moss (BM) shift [19,20], and it could make $\mathrm{ScN}$ suitable for transparent conductors demanding about a $3.0 \mathrm{eV}$ or larger optical absorption onset. The $\mathrm{BM}$ shift has also been reported in narrow-gap nitrides such as $\mathrm{InN}$ [21-23], $\mathrm{ZnSnN}_{2}$ [23], and $\mathrm{Zn}_{3} \mathrm{~N}_{2}$ [24]. In these nitrides, oxygen atoms on nitrogen sites and/or hydrogen interstitials have been considered as its origin. On the other hand, $\mathrm{N}$ vacancies, $\mathrm{O}, \mathrm{F}$, and/or Ta contamination are considered to be the main sources in $\mathrm{ScN}$ [25-27].

Native defects and foreign impurities also play crucial roles in some applications. Therefore, those in the IIIa nitrides have been extensively studied from both theoretical and experimental viewpoints, as represented by the $p$-type conversion by $\mathrm{Mg}$ doping in $\mathrm{GaN}[2,28]$. In the case of $\mathrm{ScN}$, its thermoelectric performance should depend on point-defect properties. Thus, Kerdsongpanya et al. calculated the density of state (DOS) of substitutional impurities in $\mathrm{ScN}$ using the generalized-gradient approximation (GGA) and concluded that control of the impurity concentration may improve the Seebeck coefficient [29]. Furthermore, ScN should show different defect properties than the WZ IIIa nitrides do because of differing crystal structures (a tiny interstitial space and a higher coordination number) and the differing orbital symmetry of the conduction band (CB). Therefore, the point defects in $\mathrm{ScN}$ are 
expected to raise unique properties. Stampfl et al. calculated the formation energy of the nitrogen vacancy $\left(V_{\mathrm{N}}\right)$ in the neutral charge state in bulk $\mathrm{ScN}$ and on its surface using the screened exchange local density approximation [30]. Kerdsongpanya et al. also calculated neutral $V_{\mathrm{N}}$ using the Heyd-Scuseria-Ernzerhof (HSE06) hybrid functional [29]. However, investigation into other defects is required to fully understand the defect properties in $\mathrm{ScN}$. Thus, in this paper, we calculate all possible defects, i.e., vacancies, interstitials, and antisites, in a wide range of possible charge states using the HSE06 functional. Furthermore, we carefully analyze the bulk properties (i.e., lattice parameter, Gibbs free energy of formation, band structure, and effective masses), band-edge positions, and relative chemical stability among the binary nitrides, and we discuss the unintentional doping and $p$-type dopability.

This paper is organized as follows: After describing the computational procedure and conditions in Sec. II, we provide the calculated bulk properties in Sec. III A. The optical gap as a function of the carrier-electron concentration is also compared with experimental data. We then exhibit, in Sec. III B, the band-edge positions with respect to the vacuum level along with those of the IIIa nitrides and discuss the origin of the high level of the VBM. Next, in Sec. III C, the formation energies of binary nitrides are discussed, and $\mathrm{ScN}$ is shown to be the most stable among them. Finally, calculation results on native defects, impurities, and $p$-type dopants are discussed in detail in Sec. III D.

\section{METHODS}

\section{A. Computational details}

First-principles calculations are performed using the projector augmented-wave (PAW) method [31,32], as implemented in the Vienna $a b$ initio simulation package [33]. PAW data sets with radial cutoffs of 1.32, 1.06, 1.53, $0.79,0.58,0.80,0.80$, and $0.79 \AA$ for Sc, Mg, Ta, N, H, O, F, and $\mathrm{C}$, respectively, are employed. $\mathrm{Sc} 3 s, 3 p, 4 s$, and $3 d, \mathrm{Mg}$ $3 s$, Ta $6 s$ and $5 d, \mathrm{~N} 2 s$ and $2 p, \mathrm{H} 1 s, \mathrm{C} 2 s$ and $2 p, \mathrm{O} 2 s$ and $2 p$, and $\mathrm{F} 2 s$ and $2 p$ are considered as valence electrons.

We use the HSE06 hybrid functional [34-36], which can accurately predict the atomic and electronic structures of semiconductors, especially nitrides [5,24]. Indeed, Deng et al. reported that the direct gap of $\mathrm{ScN}$ calculated by HSE06 is well matched with that determined from the optical absorption spectrum [25].

For the perfect crystals of $\mathrm{ScN}$ and WZ IIIa nitrides, lattice constants and internal atomic positions are fully optimized until the residual forces and stresses converge to less than $0.01 \mathrm{eV} / \AA$ and $0.1 \mathrm{GPa}$, respectively. The $k$-point mesh is set to a $\Gamma$-centered $8 \times 8 \times 8$ value for structure optimization of the $\mathrm{ScN}$ primitive cell, $12 \times 12 \times 12$ for dielectric constants, and $20 \times 20 \times 20$ for the DOS. For the WZ IIIa nitrides, $6 \times 6 \times 4 k$-point mesh is adopted for structure optimization. Plane-wave cutoff energy is set to $550 \mathrm{eV}$ for structure optimization and $400 \mathrm{eV}$ for the other calculations under fixed lattice constants.

\section{B. Band alignment}

Band-edge positions with respect to the vacuum level are derived from calculations of the slab-vacuum models. Here, RS (100) and (110) and WZ(112̄0) surfaces are adopted, as they belong to Tasker type I surfaces, at which the dipole should be inhibited [37-39]. The so-called macroscopically averaged potential [40] is computed by averaging the electrostatic potential within the period volume of the unit cell along the direction normal to the surfaces, and the potential difference between the bulk and vacuum regions is evaluated. In our calculations, RS (100) and (110) and WZ $(11 \overline{2} 0)$ slab models contain 32 (4 conventional unit-cell widths), 40 (5), and 24 (6) atoms, respectively, and the vacuum thickness is more than $10 \AA$. For $k$-point sampling, a $\Gamma$-centered $1 \times 6 \times 6$ mesh is adopted. The atomic positions should not be relaxed for a discussion on band alignment for avoiding the surface dipole as much as possible $[38,39]$. On the other hand, the ionization potentials calculated with atomic relaxation can be directly compared with experiments and thus will be helpful. Therefore, we show both results with and without atomic relaxation. We confirm that the macroscopically averaged potential is nearly flat in the middle of both the bulk and vacuum regions.

\section{Point defects}

The formation energy of a point defect is calculated as [41]

$$
\begin{aligned}
E_{f}\left[D^{q}\right]= & \left\{E\left[D^{q}\right]+E_{\mathrm{corr}}\left[D^{q}\right]\right\}-E_{P}-\sum n_{i} \mu_{i} \\
& +q\left(\epsilon_{\mathrm{VBM}}+\Delta \epsilon_{F}\right),
\end{aligned}
$$

where $E\left[D^{q}\right]$ and $E_{P}$ are the total energies of the supercell with defect $D$ in charge state $q$ and the perfect crystal supercell without any defect, respectively. $n_{i}$ is the number of removed $\left(n_{i}<0\right)$ or added $\left(n_{i}>0\right) i$-type atoms, and $\mu_{i}$ refers to the chemical potential. $\epsilon_{\mathrm{VBM}}$ is the energy level of the VBM, and $\Delta \epsilon_{F}$ the Fermi level $\left(\epsilon_{F}\right)$ with respect to it. Thus, $\epsilon_{F}=\epsilon_{\mathrm{VBM}}+\Delta \epsilon_{F} . E_{\mathrm{corr}}\left[D^{q}\right]$ corresponds to an energy for correcting a finite supercell size error. We adopt our extended Freysoldt-Neugebauer-Van de Walle (FNV) correction scheme, which usually estimates the defectformation energies in the dilute limit quite accurately $[24,39,41,42]$. However, as discussed in the Appendix, the FNV corrections cannot sufficiently remove the finitesize effects in $\mathrm{ScN}$, probably due to large spill out of the defect charges. Therefore, additional corrections are taken into account in this paper. Its correction energies are assumed to be proportional to $q^{2}$ and are determined from a difference of $E_{f}\left[V_{\mathrm{N}}{ }^{3+}\right]$ calculated using the 64- and 
216-atom supercells. This procedure is verified via systematic defect calculations using the Perdew-BurkeErnzerhof (PBE) GGA [43] with a Hubbard $U$ correction $(\mathrm{PBE}+U)$ (see the Appendix for details) [44]. Spin polarization is considered for all of the defects. In the defect calculations, atomic positions are fully optimized until the residual forces converge to less than $0.04 \mathrm{eV} / \AA$, whereas the lattice constants are kept fixed to the theoretical bulk values. The initial atomic positions near defects are randomly shifted up to $0.2 \AA$. The defect eigenvalues are also corrected within the point-charge approximation, as discussed in Refs. [45,46].

For point-defect calculations, Sc $3 s$ and $3 p$ orbitals are treated as core electrons so that the number of valence electrons is drastically reduced. The radial cutoff of this PAW potential is $1.59 \AA$. The errors caused by this treatment are discussed by taking nitrogen vacancies in + and $2+$ charge states as examples in Sec. III D.

\section{RESULTS AND DISCUSSION}

\section{A. Bulk properties}

Let us begin with the fundamental bulk properties listed in Table I. One can see that the theoretical lattice constant excellently agrees with the experiment, consistent with the previous report [25]. Indirect and direct gaps also agree well with the values by scanning tunneling spectroscopy [47]. The direct gap is also consistent with those evaluated from the optical absorption spectra [25,27]. For the calculation of the Gibbs free energy of formation $\left(\Delta \mathrm{G}_{f}\right)$ at the standard state, the zero phonon vibrational energy (ZPVE) and the entropic contribution are taken into account only for the $\mathrm{N}_{2}$ gas phase, as the solid phases largely tend to cancel out these two contributions near room temperature $[24,48]$.

Figure 1 shows the electronic band diagram and the density of states (DOS) projected on each element and each orbital. The band structure shows an indirect type with a minimum gap between the $\Gamma$ and $X$ points, and a minimum direct gap exists at the $X$ point, consistent with previous reports $[25,50]$. The electron and hole effective masses at

TABLE I. Lattice constant (a), band gap, and $\Delta \mathrm{G}_{f}$ at the standard state, which are obtained using HSE06, along with the experimental values (Expt.). The band gap is calculated at the theoretical lattice constant. The hexagonal close-packed (HPC) structure and the $\mathrm{N}_{2}$ gas phase are adopted as the standard states of Sc and $\mathrm{N}$, respectively. We cannot find reliable experimental data for $\Delta \mathrm{G}_{f}$.

\begin{tabular}{lcccc}
\hline \hline & & \multicolumn{2}{c}{ Minimum gap $(\mathrm{eV})$} & \\
\cline { 3 - 4 } & $a(\AA)$ & Indirect & Direct & $\Delta \mathrm{G}_{f}(\mathrm{eV} /$ atom $)$ \\
\hline Theory & 4.498 & 0.88 & 1.98 & -2.03 \\
Expt. & $4.501[49]$ & $0.9 \pm 0.1[47]$ & $2.15[47]$ & $\cdots$ \\
& \multicolumn{4}{c}{$2.07 \pm 0.05[25]$} \\
& & $2.03[27]$ \\
\hline \hline
\end{tabular}

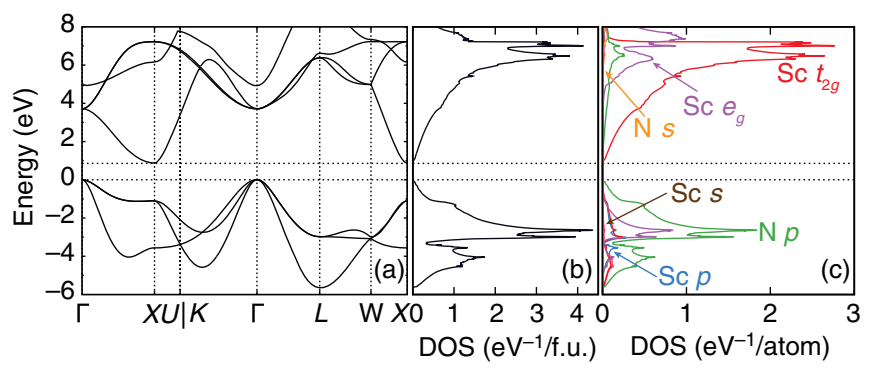

FIG. 1. (a) Band structure, (b) total DOS, and (c) site- and orbital-projected DOS calculated using HSE06 at the theoretical lattice constant. The band path is taken from Ref. [51], and the energy zero is set at the VBM.

the conduction-band minimum (CBM) and the VBM calculated from a parabolic fitting along the $X-\Gamma$ and $\Gamma-X$ lines showing the heaviest masses are $m_{e}=1.38 m_{0}$ and $m_{h}=0.81 m_{0}$, where $m_{0}$ is the mass of the free electron. Notice that $m_{h}$ is smaller than it is in GaN [5].

As shown in Fig. 1(b), Sc $3 d$ orbitals split into $t_{2 g}$ and $e_{g}$ orbitals due to crystal field splitting caused by sixfoldcoordinated nitrogen atoms. Consequently, the valence band (VB) consists mainly of $\mathrm{N} 2 p$ orbitals bonding slightly with $\mathrm{Sc} e_{g}$ orbitals, whereas the $\mathrm{CB}$ consists mainly of the nonbonding Sc $t_{2 g}$ orbitals, which are different from those in the IIIa nitrides, in which cation $n s p(n=3,4$, or 5$)$ orbitals mainly construct the CB.

Figure 2(a) shows the carrier-electron concentration as a function of the Fermi level calculated from the DOS shown in Fig. 1 along with the Fermi-Dirac distribution. These data are useful as they give the position of the Fermi level from the experimentally easily available carrier-electron concentration. Temperatures are set at 300 and $850 \mathrm{~K}$, the latter of which corresponds to a typical synthesis temperature of $\mathrm{ScN}$ [52]. Above the CBM, as electrons fill in the $\mathrm{CB}$, the temperature dependence almost disappears. This is the case because the number of excited electrons at the finite temperatures considered is much smaller than that in
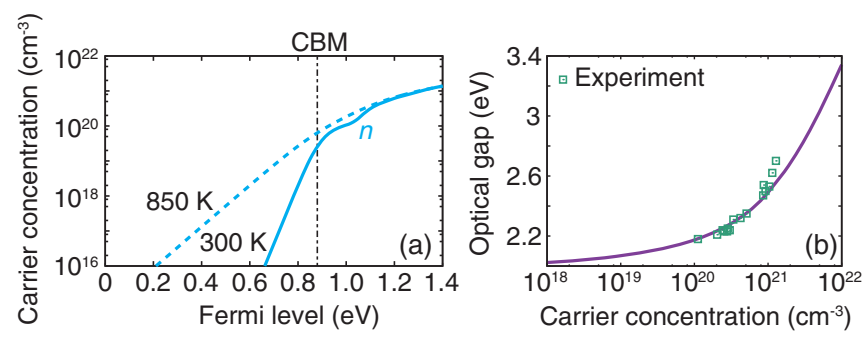

FIG. 2. (a) Carrier-electron concentration as a function of the Fermi level. Temperatures are set at room temperature (300 K) and at a typical synthesis temperature $(850 \mathrm{~K})$. (b) Optical gap as a function of the carrier-electron concentration estimated from the band structure and the DOS computed using HSE06 (see the text for details). The experimental data by Deng et al. [25] are indicated by square symbols. Notice that the carrier-electron concentration in the measured samples is controlled by doping donor-type F impurities. 
the filled $\mathrm{CB}$. We should note that the reported carrier-electron concentration in thin films ranges up to $2.0 \times 10^{21} \mathrm{~cm}^{-3}[9,10,25-27,47,52-56]$, corresponding to the Fermi level at $1.48 \mathrm{eV}$ at $300 \mathrm{~K}$.

Now, we can quantify the optical gap as a function of the carrier-electron concentration from Figs. 1(a) and 1(b), as shown in Fig. 2(b). The experimental values measured for F-doped samples [25] are also shown. Here, the optical onset corresponds to the minimum direct gap from the occupied to the unoccupied state in the band structure. One can see that a curve of the theoretical gap amazingly coincides with the experimental values, indicating that the HSE06 electronic structure is very close to the experimental one. It has also been reported in experiments that the introduction of holes does not largely change the optical gap in $\mathrm{ScN}$ [56], which is also consistent with our calculation showing that the minimum direct gap locates at the $X$ point, and the VBM at the $\Gamma$ point.

\section{B. Band alignment}

The band-edge positions of $\mathrm{ScN}$ are aligned in Fig. 3 alongside those of the IIIa nitrides for comparison. Our computed positions of the IIIa nitrides are very close to the values reported by Moses et al. [57]. As expected, the VBM positions at the (100) and (110) surfaces are very close to each other as they belong to the Tasker type I surfaces $[37,38,58]$. The VBMs in $\mathrm{ScN}$ are relatively much higher even compared to that in $\mathrm{InN}$ with a similar band gap. This tendency is presumably attributable to the difference in coordination number; a higher coordination number leads

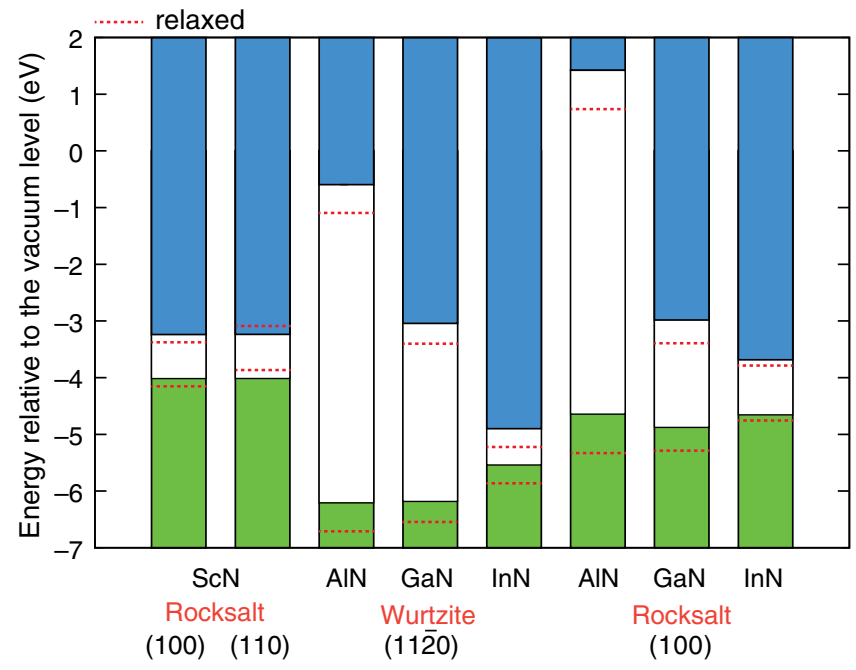

FIG. 3. Band-edge positions of $\mathrm{ScN}$ and the WZ IIIa nitrides with respect to the vacuum level calculated using HSE06 at the theoretical lattice constants. To discuss the structure dependences, the hypothetical RS IIIa nitrides are also calculated. Note that the RS (100) and (110) surfaces and the WZ(1120) surface are classified into the Tasker type I surfaces. Results both with and without atomic relaxation in the slab models are shown. The former is plotted with red dotted lines, the latter with solid lines. to larger orbital interactions, i.e., larger band dispersion. As a result, $\mathrm{ScN}$ exhibits the higher VBM and the smaller hole effective mass $\left(m_{h}=0.81 m_{0}\right)$. Indeed, the VBM positions of the IIIa nitrides in the RS structure are higher by $0.9-1.6 \mathrm{eV}$ than those in the $\mathrm{WZ}$ structure, as shown in Fig. 3. The remaining contribution may be covalent bonding of N $2 p$ orbitals with Sc $3 d$ orbitals.

When the atomic relaxation is considered, the band-edge positions are mostly moved down. The same tendencies for IIIa nitrides were reported by Moses et al. [57].

\section{Chemical stability}

In this subsection, the chemical stability of $\mathrm{ScN}$ is compared with other binary nitrides using the entries in the Materials Project (MP) database [59,60]. We compile the formation energies of binary nitrides that are stable with respect to the competing phases in Fig. 4(a). As the MP database is constructed mainly using PBE, we calculate the formation energies of IIIb (Sc, Y, and Lu) and IIIa (Al, Ga, and In) nitrides using the HSE06 functional for comparison. As shown in Fig. 4(a), it is found that the sequences of the stability are the same, but the formation energiesespecially for InN and LuN-are largely different. Such a discrepancy is mainly attributed to the empirical correction on N-N binding energy in the MP database, and the differences of cohesive energies of metallic substances. Intriguingly, rare-earth nitrides are fairly stable; especially
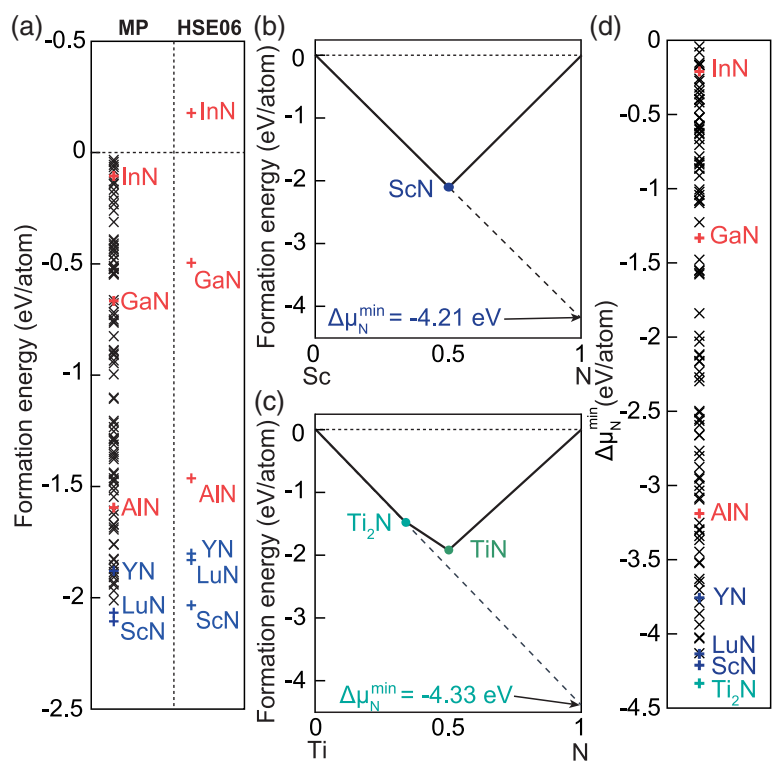

FIG. 4. (a) Formation energies of 95 binary nitrides and (d) their $\mathrm{N}$ chemical potentials at the $\mathrm{N}$-poor condition $\left(\Delta \mu_{\mathrm{N}}^{\min }\right)$. Data are extracted from the Materials Project (MP) database, which uses PBE [59,60]. Unstable compounds in the MP database are excluded from this plot. For comparison, we also plot the formation energies of IIIb (Sc, Y, and Lu) and IIIa (Al, Ga, and In) nitrides calculated using the HSE06 functional. (b) and (c) exemplify how to determine $\mu_{\mathrm{N}}$ at the $\mathrm{N}$-poor condition. 
$\mathrm{ScN}$ and $\mathrm{LuN}$ are the most and the secondmost stable, respectively. Such very high stability should be connected to the very high melting point and mechanical hardness. On the contrary, the IIIa nitrides are scattered widely and $\mathrm{InN}$ shows a nearly zero formation energy.

Once the convex hulls are depicted, we can calculate the limit of the nitrogen chemical potentials $\left(\mu_{\mathrm{N}}\right)$ from the convex hulls, as exemplified in Figs. 4(b) and 4(c). We show $\mu_{\mathrm{N}}$ of these binary nitrides at the $\mathrm{N}$-poor condition in Fig. 4(d). Generally speaking, as $\mu_{\mathrm{N}}$ decreases, the formation energy of $V_{\mathrm{N}}$ decreases. As seen in Fig. $4(\mathrm{~d}), \mathrm{Ti}_{2} \mathrm{~N}$ attains the lowest $\mu_{\mathrm{N}}$ value, yet is a metal. $\mathrm{ScN}$ is the second lowest and shows the lowest $\mu_{\mathrm{N}}$ value among the binary nitrides with band gaps. The large controllability of $\mu_{\mathrm{N}}$ should be part of the reason why $\mathrm{ScN}$ shows the BM shift even with such a high CBM position, as shown in Fig. 3, and attains the $p$-type conversion on the other hand.

\section{Point defects}

Here, we show the calculated defect-formation energies under Sc- and N-rich conditions. Thermodynamic equilibrium conditions are assumed to be satisfied in this paper. The calculated phase diagrams are compiled in the Appendix. We note here that a Sc-rich condition with $\mu_{\mathrm{N}}=$ $-4.05 \mathrm{eV}$ is achieved at a very low nitrogen chemical pressure of $p_{\mathrm{N}_{2}}=10^{-19} \mathrm{~Pa}$ at $1200 \mathrm{~K}$, which are not easily attained. Therefore, the experimental conditions usually exist between these two conditions.

As described in Sec. II, we use for defect calculations the Sc PAW potential that does not explicitly treat Sc $3 s p$ orbitals as valence electrons. The lattice constant and the band gap of $\mathrm{ScN}$ are then slightly underestimated, from 4.498 to $4.479 \AA$ and from 0.88 to $0.78 \mathrm{eV}$, respectively. To examine this treatment, we calculate $V_{\mathrm{N}}^{+}$and $V_{\mathrm{N}}{ }^{2+}$, which have the lowest formation energies among the native defects; differences of $E\left[V_{\mathrm{N}}^{+}\right]$and $E\left[V_{\mathrm{N}}{ }^{2+}\right]$ calculated using two different Sc PAW potentials are only 0.05 and $0.06 \mathrm{eV}$, respectively, when the Fermi level is located at the VBM.

For reciprocal space integration, we use the $2 \times 2 \times 2$ Monkhorst-Pack $k$-point mesh, which excludes the band edges at the $\Gamma$ and $X$ points. The CBM is then spuriously raised by $1.0 \mathrm{eV}$ within the sampled $k$ points, so the defect levels are properly calculated up to that point [24]. Therefore, the range of $\Delta \epsilon_{F}$ considered is $0-1.4 \mathrm{eV}$, which almost covers the Fermi level and carrier-electron concentration observed in the experiments (see Figs. 2 and 3).

\section{Native defects}

Figures 5(a) and 5(b) show the formation energies of the native defects in $\mathrm{ScN}$ as a function of $\Delta \epsilon_{F}$. Noticeably, $V_{\mathrm{Sc}}$ is the only acceptor-type defect when $\Delta \epsilon_{F}$ is varied within the band gap, but it has a very high formation energy even at the N-rich condition. Conversely, $V_{\mathrm{N}}$ is known to show much lower formation energies in some nitrides. Indeed, at

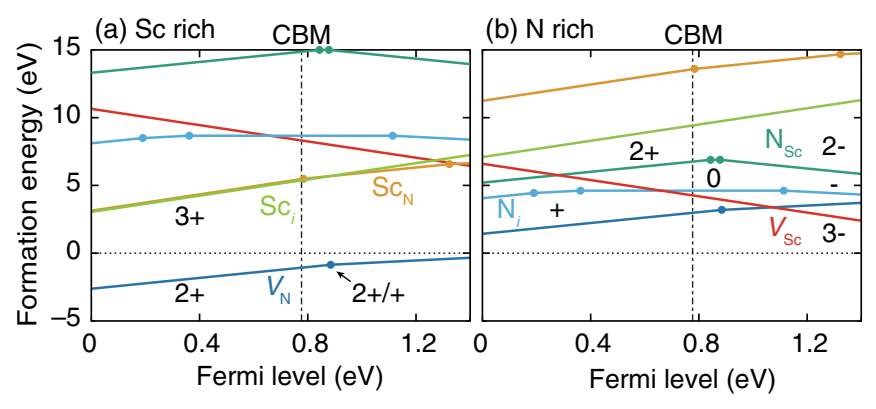

FIG. 5. Formation energies of native point defects in $\mathrm{ScN}$ under (a) Sc-rich and (b) N-rich conditions as a function of the Fermi level. The energy zero is set at the VBM, and the CBM is designated by the dashed lines. The defect species and sites are indicated by $X_{Y}$, where $X$ is the vacancy $(V)$ or element and $Y$ is the defect site ( $i$ means an interstitial site at the center of the tetrahedral site).

the Sc-rich condition, $E\left[V_{\mathrm{N}}\right]$ is negative at an entire Fermi level and should generate a significant number of carrier electrons. However, at the N-rich condition, $V_{\mathrm{N}}$ shows a positive formation energy and does not cause a Fermi-level pinning within the band gap. Therefore, $p$-type doping is attainable as long as carrier compensation by unintentional impurities is well suppressed, and suitable dopants are present (see Sec. III D3). Such a large variation in the defect-formation energies stems from the very high controllability of the chemical potentials in $\mathrm{ScN}$, as discussed in Sec. III C.

The defect states caused by $V_{\mathrm{N}}$ are composed mainly of Sc $e_{g}$ orbitals (see Fig. 6). Intriguingly, only $V_{\mathrm{N}}{ }^{2+}$ emerges in the band gap, which is not so stable compared to + and $3+$ charge states in the IIIa nitrides $[24,62,63]$. When the localized $d$ orbitals constitute mainly the defect state, as $V_{\mathrm{N}}$ in $\mathrm{ScN}$, the on-site Coulomb repulsion is expected to be large. Consequently, $V_{\mathrm{N}}$ preferentially accommodates only one electron, which would be the reason why $V_{\mathrm{N}}{ }^{2+}$ is relatively stable in $\mathrm{ScN}$. Indeed, as shown in Fig. 6, when

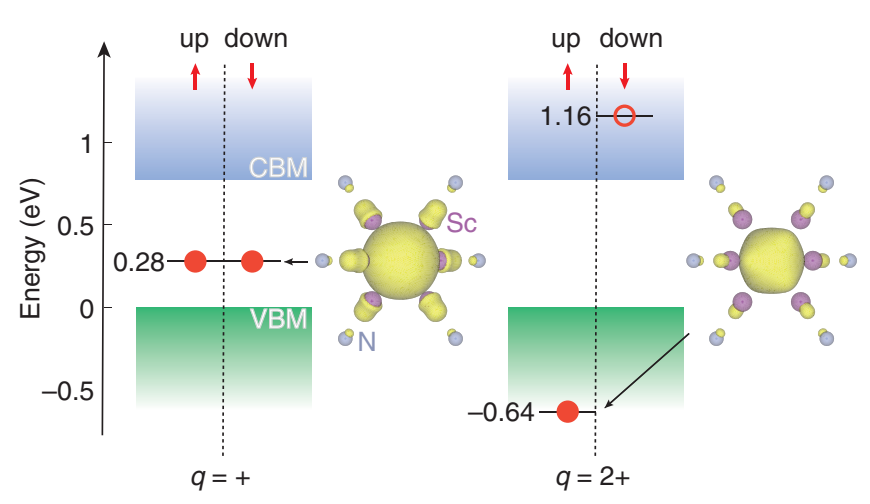

FIG. 6. Spin-resolved single-particle levels of $V_{\mathrm{N}}$. The isosurfaces of the squared wave functions for the localized defect states are also shown; they are visualized using VESTA [61]. 
accommodating one electron, the occupied defect level is buried in the VB, whereas the unoccupied one is above the $\mathrm{CBM}$. This discussion is analogous to the shallow behavior of oxygen vacancies in perovskite titanates such as $\mathrm{SrTiO}_{3}$ having a similar electronic structure $[64,65]$.

The formation energies of the interstitials are very high, probably due to tiny tetrahedral space. Similarly, the antisite defects are also very high in energy and are not so important. However, it is still worth noting that $\mathrm{N}_{\mathrm{Sc}}$ and $\mathrm{N}_{i}$ show various types of defect structures, depending on the Fermi level. Although some of them are already known in other nitrides, such as a $\mathrm{N}_{2}$ moleculelike structure for $\mathrm{N}_{i}$ in $\mathrm{GaN}$ [66] and a $\mathrm{N}_{3}$ azidelike structure for $\mathrm{N}_{\mathrm{Zn}}$ in $\mathrm{Zn}_{3} \mathrm{~N}_{2}$ [24], we newly find a $\mathrm{N}_{4}$ cluster structure for $\mathrm{N}_{\mathrm{Sc}}{ }^{2+}$.

\section{Impurities}

Figure 7 exhibits the formation energies of unintentionally introduced impurities: $\mathrm{H}, \mathrm{O}, \mathrm{F}$, and Ta at the substitutional sites and $\mathrm{H}$ and $\mathrm{O}$ at the interstitial site. In the experiments, $\mathrm{H}$ and $\mathrm{O}$ are well known to be unintentionally introduced from the air into nitrides, whereas $\mathrm{F}$ and $\mathrm{Ta}$ potentially contaminate into $\mathrm{ScN}$ during the refining process [25]. Some of them are considered to be main sources of the BM shift in $\mathrm{ScN}$. The chemical potentials are determined from the thermodynamic equilibrium conditions as shown in the Appendix. These conditions give the lower limits of the formation energies and, therefore, the solubility limits of the impurities.

The O-on- $\mathrm{N}\left(\mathrm{O}_{\mathrm{N}}\right)$ impurity acts as a single donor, and the $\mathrm{H}$-on-N $\left(\mathrm{H}_{\mathrm{N}}\right)$ impurity forms a multicenter bonding with six Sc neighbors and acts as a double donor, as with other nitrides $[24,63]$. At the $\mathrm{N}$-rich condition, $\mathrm{H}$ preferentially locates at an interstitial site near $\mathrm{N}$ with the $\mathrm{N}-\mathrm{H}$ bond distance of $1.03 \AA$, which is close to that of the $\mathrm{NH}_{3}$ molecule (1.01 $\AA$ by HSE06). On the other hand, $\mathrm{H}_{\mathrm{N}}^{2-}$ is stable at the Sc-rich condition, indicating $\mathrm{H}$ mostly exists as a hydride ion, which is unusual in other nitrides [see the inset in Fig. 7(a)]. As $\mathrm{H}_{\mathrm{N}}$ shows a very low formation energy, such multicenter bonding states should be detectable in $\mathrm{ScN}$. $\mathrm{F}_{\mathrm{N}}$ is a double donor, consistent with

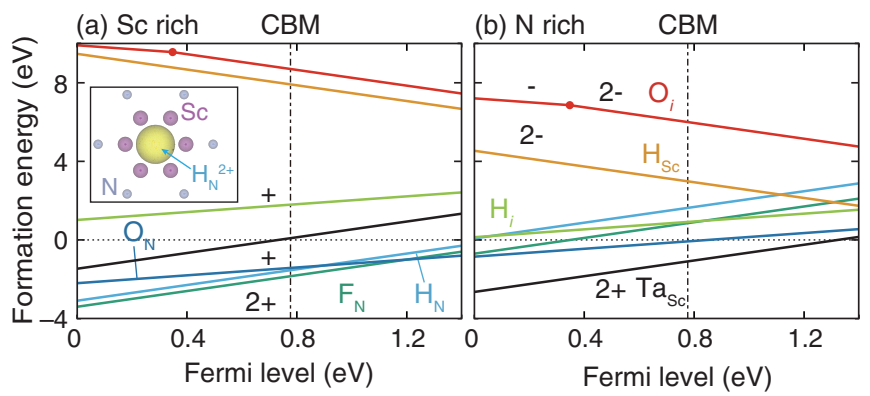

FIG. 7. The same as Fig. 5, but for H, O, F, and Ta impurities. The isosurface of the squared wave function for the hydrogen state at $\mathrm{H}_{\mathrm{N}}^{2+}$ are also shown. the fact that $\mathrm{F}$ doping introduces carrier electrons up to $1.3 \times 10^{21} \mathrm{~cm}^{-3}$ [25]. For the application as transparent conductors, we need to introduce more carrier electrons to increase the optical absorption onset. As $E_{f}\left[\mathrm{O}_{\mathrm{N}}^{+}\right]$is lower than $E_{f}\left[\mathrm{~F}_{\mathrm{N}}{ }^{2+}\right]$ at a Fermi level higher than $1.2 \mathrm{eV}$, oxygen doping may be suited for this purpose. $\mathrm{Ta}_{\mathrm{Sc}}$ is also a double donor and shows a very low formation energy, especially at the N-rich condition. On the contrary, the acceptor-type defects, i.e., $\mathrm{O}_{i}$ and $\mathrm{H}_{\mathrm{Sc}}$ are not stable within the range of the Fermi level considered. Based on our calculations, the origins of the $\mathrm{BM}$ shift would be $\mathrm{H}_{\mathrm{N}}, \mathrm{F}_{\mathrm{N}}$, and $\mathrm{O}_{\mathrm{N}}$ at the Sc-rich condition, and $\mathrm{O}_{\mathrm{N}}$ and $\mathrm{Ta}_{\mathrm{Sc}}$ at the $\mathrm{N}$-rich condition. Notice again that $V_{\mathrm{N}}$ can also be a main source of the BM shift at the cation-rich condition, which is different from InN [63] and $\mathrm{Zn}_{3} \mathrm{~N}_{2}$ [24].

\section{3. p-type doping}

We now discuss the possibility of $p$-type doping. For acceptor dopants, we select $\mathrm{Mg}$ and $\mathrm{C}$, which are supposed to occupy the Sc site and the $\mathrm{N}$ site, respectively. Note that the ionic radius of $\mathrm{Mg}(0.86 \AA)$ is very close to that of Sc $(0.885 \AA)$ [67]. Here, we consider only the N-rich condition because $V_{\mathrm{N}}$ is positive in energy inside the gap. As discussed in Sec. III D 2, the contamination by O, Ta, and $\mathrm{F}$ that kills holes should be avoided as much as possible.

The calculated formation energies of the substituted $\mathrm{Mg}$ and $\mathrm{C}$ acceptor dopants are shown in Fig. 8. One can see that $\mathrm{Mg}_{\mathrm{Sc}}$ shows a shallow acceptor behavior and $\mathrm{C}_{\mathrm{N}}$ shows a slightly deep state. These findings are in contrast with $\mathrm{Mg}_{\mathrm{Ga}}$ [28] and $\mathrm{C}_{\mathrm{N}}[68$ ] in $\mathrm{GaN}$, showing much deeper states. These differences are examined in terms of the difference of the VBM positions (see Fig. 3). Furthermore, $\mathrm{Mg}_{\mathrm{Sc}}$ is lower in energy than $V_{\mathrm{N}}$ in the band gap. Therefore, $\mathrm{Mg}$ should be a good acceptor dopant for $\mathrm{ScN}$. Indeed, very recently, Saha et al. synthesized $p$-type $\mathrm{ScN}$ with $\mathrm{Mg}$ doping with a hole concentration up to $2 \times 10^{20} \mathrm{~cm}^{-3}$ [56]. They did not find any deep defect states inside the gap by $\mathrm{Mg}$ doping, which is again consistent with the shallow acceptor behavior found in this paper.

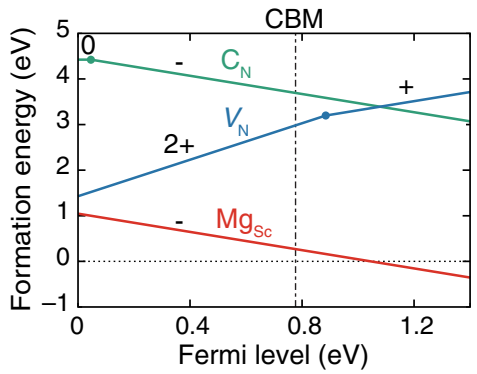

FIG. 8. The same as Fig. 5, but for $\mathrm{Mg}$ and $\mathrm{C}$ dopants at the $\mathrm{N}$-rich condition. The energy of $V_{\mathrm{N}}$ is also shown for comparison. 


\section{CONCLUSIONS}

We investigate in this work the bulk and point-defect properties of $\mathrm{ScN}$ using HSE06. We first calculate the lattice constant, band structure, DOS, and Gibbs free energy of formation at the standard state in Sec. III A. The calculated lattice constant and the band gap agree excellently with the experiments. Regarding the electronic structure, the VB and the $\mathrm{CB}$ are mainly composed of the $\mathrm{N}$ $2 p$ orbitals and the nonbonding Sc $t_{2 g}$ orbitals, respectively. The calculated effective masses are $m_{e}=1.38 m_{0}$ and $m_{h}=0.81 m_{0}$. We then depict the optical gap as a function of the carrier-electron concentration, and we find that the theoretical curve agrees well with the experimental data. This result indicates that the HSE06 electronic structure is very close to the experimental one.

In Sec. III B, we discuss the band-edge positions of $\mathrm{ScN}$ relative to those of the IIIa nitrides. The VBM of $\mathrm{ScN}$ is much higher than those of the IIIa nitrides. This tendency can be mainly attributed to the difference in the coordination number. Indeed, the VBM positions of the IIIa nitrides are increased by $0.9-1.6 \mathrm{eV}$ if they are in the RS structure.

In Sec. III C, the chemical stability of $\mathrm{ScN}$ is compared to other binary nitrides using the entries in the Materials Project $[59,60]$. ScN is found to be the most stable among the binary nitrides. We also find that $\mathrm{ScN}$ can reach the lowest nitrogen chemical potential among the binary nitrides with band gaps.

In Sec. III D, we show the calculated formation energies of the native defects, unintentionally introduced impurities, and $p$-type dopants. We find that $V_{\mathrm{Sc}}$ is the only acceptortype defect when the Fermi level is located within the band gap, but it has a very high formation energy even at the $\mathrm{N}$-rich condition. Conversely, at the Sc-rich condition, the energy of $V_{\mathrm{N}}$ is negative at an entire Fermi-level range and should introduce a significant number of carrier electrons. On the contrary, at the $\mathrm{N}$-rich condition, $V_{\mathrm{N}}$ shows a positive formation energy for the Fermi-level position within the band gap. Regarding the interstitials and antisites, their formation energies are very high, but some peculiar structures are found, such as the $\mathrm{N}_{4}$ cluster structure for $\mathrm{N}_{\mathrm{Sc}}^{2+}$. Among the unintentionally introduced impurities, the $\mathrm{O}_{\mathrm{N}}$ impurity acts as a single donor, and the $\mathrm{H}_{\mathrm{N}}$ impurity forms a multicenter bonding, as it is a double donor. At the $\mathrm{N}$-rich condition, $\mathrm{H}$ preferentially locates at an interstitial site near $\mathrm{N}$, but $\mathrm{H}_{\mathrm{N}}$ is stable at the Sc-rich condition. $\mathrm{F}_{\mathrm{N}}$ and $\mathrm{Ta}_{\mathrm{Sc}}$ are double donors and show very low formation energies that depend on the growth condition. Based on our calculations, the origins of the BM shift would be $V_{\mathrm{N}}, \mathrm{H}_{\mathrm{N}}, \mathrm{F}_{\mathrm{N}}$, and $\mathrm{O}_{\mathrm{N}}$ at the $\mathrm{Sc}$-rich condition, and $\mathrm{O}_{\mathrm{N}}$ and $\mathrm{Ta}_{\mathrm{Sc}}$ at the $\mathrm{N}$-rich condition. Regarding the $p$-type conversion, $\mathrm{Mg}$ doping at the $\mathrm{N}$-rich condition should be a good choice, which is also consistent with a very recent experimental report [56].

\section{ACKNOWLEDGMENTS}

This work was supported by the MEXT Elements Strategy Initiative to Form Core Research Center, Grants-in-Aid for Young Scientists A (Grant No. 15H05541) and Scientific Research A (Grant No. 17H01318) from JSPS, and PRESTO (JPMJPR16N4) and the Support Program for Starting Up Innovation Hub $\mathrm{MI}^{2}$ I from JST, Japan.

Y. K. and N. T. contributed equally to this work.

\section{APPENDIX: COMPUTATIONAL DETAILS}

\section{Phase diagrams}

Here, we provide the ternary phase diagrams used for the calculations of impurities and dopants in Fig. 9. The Sc-rich and N-rich conditions used are also highlighted by circles. Note that all of the calculations are performed using the HSE06 functional, and the ZPVE and entropic contributions are taken into account only for the gas phases.
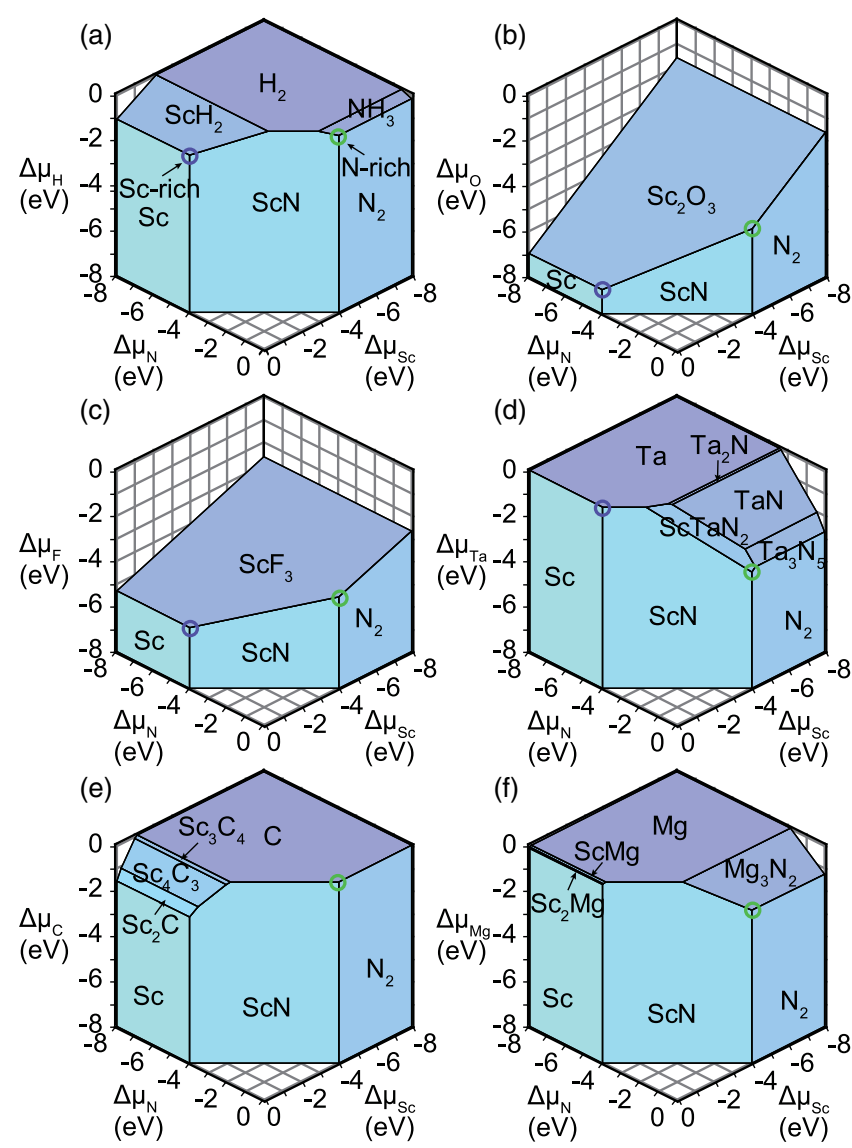

FIG. 9. Chemical potential diagrams for the Sc-N-X $(X=\mathrm{H}, \mathrm{O}$, $\mathrm{F}, \mathrm{Ta}, \mathrm{C}$, and $\mathrm{Mg}$ ) ternary systems visualized with CHESTA [69]. The standard states are set to the gas phases of $\mathrm{N}_{2}, \mathrm{H}_{2}, \mathrm{O}_{2}$, and $\mathrm{F}_{2}$, and HPC Sc, graphite $\mathrm{C}$, cubic Ta, and $\mathrm{HPC} \mathrm{Mg}$. The equilibrium points used for calculating defect-formation energies are designated by blue and green circles, corresponding to the Sc-rich and $\mathrm{N}$-rich conditions, respectively. 


\section{Energy corrections for point defects}

Figure 10 shows the relative formation energies of $V_{\mathrm{Sc}}{ }^{3-}$, $\mathrm{H}_{\mathrm{Sc}}{ }^{2-}, \mathrm{Mg}_{\mathrm{Sc}}^{-}, \mathrm{O}_{\mathrm{N}}^{+}, \mathrm{F}_{\mathrm{N}}{ }^{2+}$, and $V_{\mathrm{N}}^{3+}$ with no corrections and extended FNV corrections as a function of the supercell size. The calculations are performed using $\mathrm{PBE}+U$ $\left(U_{\text {eff }}=3.5 \mathrm{eV}\right)$, which leads to the band gap of $0.74 \mathrm{eV}$. The extended FNV corrections are performed using the dielectric constant in the long-range limit, namely, the sum of the electronic and ionic dielectric constants. The calculated electronic $\left(\epsilon_{\text {ele }}\right)$ and ionic $\left(\epsilon_{\text {ion }}\right)$ dielectric constants are 9.0 and 20.2 using $\mathrm{PBE}+U$. In the main text, we use $\epsilon_{\text {ele }}=8.8$ and $\epsilon_{\text {ion }}=14.6$ calculated at the HSE06 lattice constant, where $\epsilon_{\text {ele }}$ is estimated using HSE06, and $\epsilon_{\text {ion }}$ using $\mathrm{PBE}+U$.
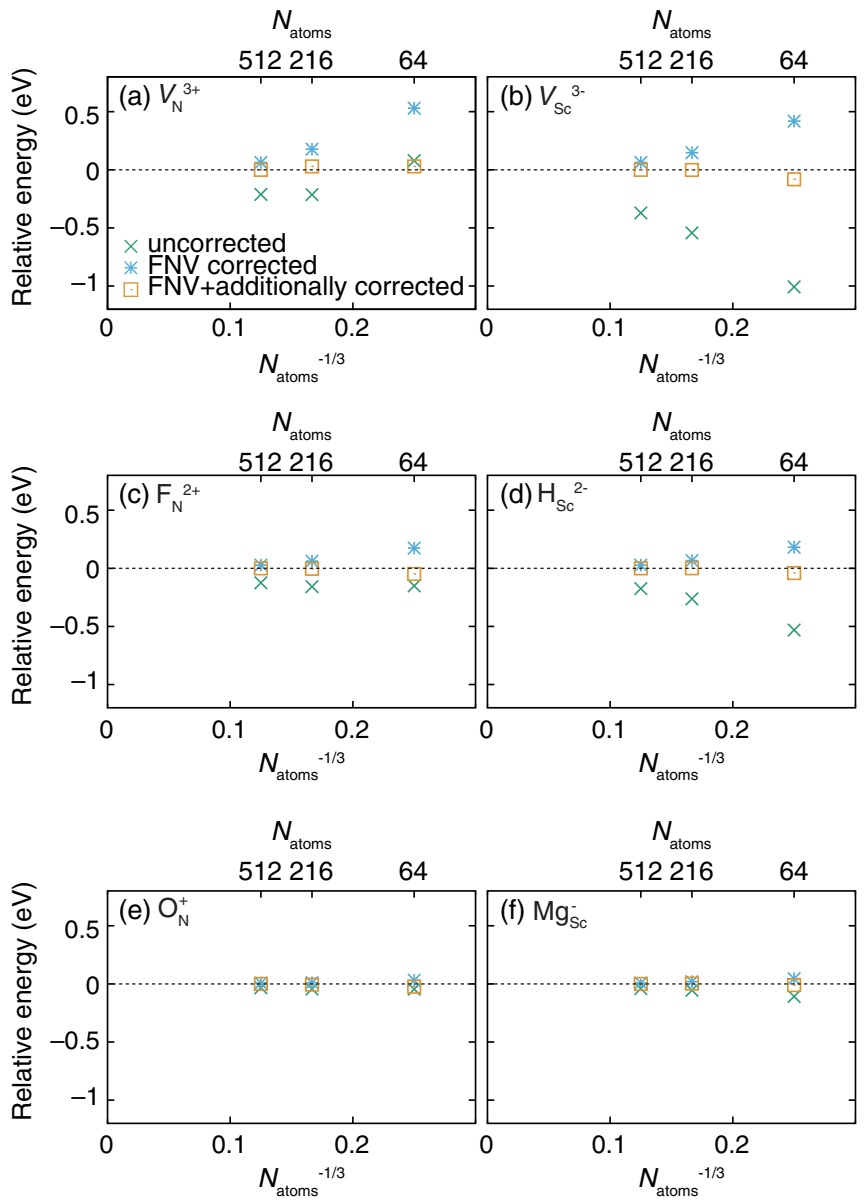

FIG. 10. Uncorrected, extended FNV corrected, and additionally corrected defect-formation energies as a function of the supercell size using $\mathrm{PBE}+U\left(U_{\text {eff }}=3.5 \mathrm{eV}\right) . N_{\text {atom }}$ is the number of atoms in the supercell before introducing a defect. The supercells are based on the expansion of the conventional cell. In order to evaluate the absolute errors, the ranges of $y$ axes are standardized. All of the calculations are performed with the $2 \times 2 \times 2$ Monkhorst-Pack $k$-point mesh for minimizing the sampling errors. The zeros are set at the energies with additional corrections at the largest supercell considered, i.e., the 512-atom supercell.
As shown, the convergences are rather slow, especially for defects with large absolute charge states. The reasons of such slow convergences are unclear but probably due to large spill out of the defect charges. To remedy the slow convergences, we further apply corrections to the 64-atom supercell results on top of the FNV corrections. As shown in Fig. 10, the cell-size dependences are roughly proportional to $q^{2}$. Therefore, we assume that the FNV corrected energies are fitted by using the following equation:

$$
E_{f}^{\mathrm{FNV}}\left[D^{q}\right]=q^{2} a N_{\mathrm{atom}}^{-1}+b\left[D^{q}\right],
$$

where $N_{\text {atom }}$ is the number of atoms in the supercell before introducing a defect and is thus proportional to the supercell volume. The fitting parameter $a$ is irrelevant to the defect species, and $b\left[D^{q}\right]$ corresponds to the energy of $D^{q}$ at the dilute limit. In this paper, we determine $a$ from $E_{f}^{\mathrm{FNV}}\left[V_{\mathrm{N}}{ }^{3+}\right]$, calculated using the 64- and 216-atom supercells, because $V_{\mathrm{N}}$ is the most important native defect and its $3+$ charge state shows the largest error in this paper. The calculated $a$ value is $3.56 \mathrm{eV} / e^{2}$ when using the $\mathrm{PBE}+U$, where $e$ represents the elementary charge. Once $a$ is obtained, we can estimate the additional correction energies by subtracting $q^{2} a N_{\text {atom }}^{-1}$.

Such additional corrections are verified with the six types of defects shown in Fig. 10; the energies of all of the defects, including $V_{\mathrm{Sc}}{ }^{3+}$, are drastically improved, and the remaining errors for the 64-atom supercell results are reduced to less than $0.08 \mathrm{eV}$, indicating that these corrections work quite well. We determine the parameters $a$ in the same way based on the HSE06 calculations $\left(a=5.70 \mathrm{eV} / e^{2}\right)$, and we use it for the corrections of all of the defect calculations with the 64-atom supercell in the main text.

[1] I. Akasaki, Nobel lecture: Fascinated journeys into blue light, Rev. Mod. Phys. 87, 1119 (2015).

[2] H. Amano, Nobel lecture: Growth of GaN on sapphire via low-temperature deposited buffer layer and realization of p-type $\mathrm{GaN}$ by $\mathrm{Mg}$ doping followed by low-energy electron beam irradiation, Rev. Mod. Phys. 87, 1133 (2015).

[3] S. Nakamura, Nobel lecture: Background story of the invention of efficient blue ingan light emitting diodes, Rev. Mod. Phys. 87, 1139 (2015).

[4] Y. Taniyasu, M. Kasu, and T. Makimoto, An aluminium nitride light-emitting diode with a wavelength of 210 nanometres, Nature (London) 441, 325 (2006).

[5] Y. Hinuma, T. Hatakeyama, Y. Kumagai, L. A. Burton, H. Sato, Y. Muraba, S. Iimura, H. Hiramatsu, I. Tanaka, H. Hosono, and F. Oba, Discovery of earth-abundant nitride semiconductors by computational screening and highpressure synthesis, Nat. Commun. 7, 11962 (2016).

[6] A. Zakutayev, Design of nitride semiconductors for solar energy conversion, J. Mater. Chem. A 4, 6742 (2016). 
[7] C. T. Horovitz, J. K. A. Gschneidner, G. A. Melson, D. H. Youngblood, and H. H. Schock, Scandium: Its Occurrence, Chemistry Physics, Metallurgy, Biology and Technology (Academic Press, New York, 1975).

[8] D. Gall, I. Petrov, N. Hellgren, L. Hultman, J. E. Sundgren, and J. E. Greene, Growth of poly- and single-crystal ScN on $\mathrm{MgO}(001)$ : Role of low-energy $\mathrm{N}_{2}^{+}$irradiation in determining texture, microstructure evolution, and mechanical properties, J. Appl. Phys. 84, 6034 (1998).

[9] S. Kerdsongpanya, N. V. Nong, N. Pryds, A. Žukauskaitė, J. Jensen, J. Birch, J. Lu, L. Hultman, G. Wingqvist, and P. Eklund, Anomalously high thermoelectric power factor in epitaxial ScN thin films, Appl. Phys. Lett. 99, 232113 (2011).

[10] P. V. Burmistrova, J. Maassen, T. Favaloro, B. Saha, S. Salamat, Y. R. Koh, M. S. Lundstrom, A. Shakouri, and T. D. Sands, Thermoelectric properties of epitaxial $\mathrm{ScN}$ films deposited by reactive magnetron sputtering onto MgO(001) substrates, J. Appl. Phys. 113, 153704 (2013).

[11] A. Herwadkar and W. R. L. Lambrecht, Mn-doped ScN: A dilute ferromagnetic semiconductor with local exchange coupling, Phys. Rev. B 72, 235207 (2005).

[12] M. Akiyama, T. Kamohara, K. Kano, A. Teshigahara, Y. Takeuchi, and N. Kawahara, Enhancement of piezoelectric response in scandium aluminum nitride alloy thin films prepared by dual reactive cosputtering, Adv. Mater. 21, 593 (2009).

[13] G. Wingqvist, F. Tasndi, A. Zukauskaite, J. Birch, H. Arwin, and L. Hultman, Increased electromechanical coupling in $w-\mathrm{Sc}_{x} \mathrm{Al}_{1-x} \mathrm{~N}$, Appl. Phys. Lett. 97, 112902 (2010).

[14] R. Matloub, M. Hadad, A. Mazzalai, N. Chidambaram, G. Moulard, C. S. Sandu, Th. Metzger, and P. Muralt, Piezoelectric $\mathrm{Al}_{1-x} \mathrm{Sc}_{x} \mathrm{~N}$ thin films: A semiconductor compatible solution for mechanical energy harvesting and sensors, Appl. Phys. Lett. 102, 152903 (2013).

[15] F. Tasnádi, B. Alling, C. Höglund, G. Wingqvist, J. Birch, L. Hultman, and I. A. Abrikosov, Origin of the Anomalous Piezoelectric Response in Wurtzite $\mathrm{Sc}_{x} \mathrm{Al}_{1-x} \mathrm{~N}$ Alloys, Phys. Rev. Lett. 104, 137601 (2010).

[16] M. E. Little and M. E. Kordesch, Band-gap engineering in sputter-deposited $\mathrm{Sc}_{x} \mathrm{Ga}_{1-x} \mathrm{~N}$, Appl. Phys. Lett. 78, 2891 (2001).

[17] C. Constantin, H. Al-Brithen, M. B. Haider, D. Ingram, and A. R. Smith, ScGaN alloy growth by molecular beam epitaxy: Evidence for a metastable layered hexagonal phase, Phys. Rev. B 70, 193309 (2004).

[18] R. Deng, P. Y. Zheng, and D. Gall, Optical and electron transport properties of rock-salt $\mathrm{Sc}_{1-x} \mathrm{Al}_{x} \mathrm{~N}$, J. Appl. Phys. 118, 015706 (2015).

[19] E. Burstein, Anomalous optical absorption limit in InSb, Phys. Rev. 93, 632 (1954).

[20] T. S. Moss, The interpretation of the properties of indium antimonide, Proc. Phys. Soc. London Sect. B 67, 775 (1954).

[21] J. Wu, W. Walukiewicz, S. X. Li, R. Armitage, J. C. Ho, E. R. Weber, E. E. Haller, H. Lu, W. J. Schaff, A. Barcz, and R. Jakiela, Effects of electron concentration on the optical absorption edge of InN, Appl. Phys. Lett. 84, 2805 (2004).

[22] P. Rinke, M. Scheffler, A. Qteish, M. Winkelnkemper, D. Bimberg, and J. Neugebauer, Band gap and band parameters of $\mathrm{InN}$ and $\mathrm{GaN}$ from quasiparticle energy calculations based on exact-exchange density-functional theory, Appl. Phys. Lett. 89, 161919 (2006).

[23] L. Lahourcade, N. C. Coronel, K. T. Delaney, S. K. Shukla, N. A. Spaldin, and H. A. Atwater, Structural and optoelectronic characterization of RF sputtered $\mathrm{ZnSnN}_{2}$, Adv. Mater. 25, 2562 (2013).

[24] Y. Kumagai, K. Harada, H. Akamatsu, K. Matsuzaki, and F. Oba, Carrier-Induced Band-Gap Variation and Point Defects in $\mathrm{Zn}_{3} \mathrm{~N}_{2}$ from First Principles, Phys. Rev. Applied 8, 014015 (2017).

[25] R. Deng, B. D. Ozsdolay, P. Y. Zheng, S. V. Khare, and D. Gall, Optical and transport measurement and first-principles determination of the $\mathrm{ScN}$ band gap, Phys. Rev. B 91, 045104 (2015).

[26] A. R. Smith, H. A. H. AL-Brithen, D. C. Ingram, and D. Gall, Molecular beam epitaxy control of the structural, optical, and electronic properties of $\mathrm{ScN}(001)$, J. Appl. Phys. 90, 1809 (2001).

[27] B. Saha, G. Naik, V. P. Drachev, A. Boltasseva, E. E. Marinero, and T. D. Sands, Electronic and optical properties of $\mathrm{ScN}$ and $(\mathrm{Sc}, \mathrm{Mn}) \mathrm{N}$ thin films deposited by reactive DCmagnetron sputtering, J. Appl. Phys. 114, 063519 (2013).

[28] J. L. Lyons, A. Janotti, and C. G. Van de Walle, Shallow versus Deep Nature of $\mathrm{Mg}$ Acceptors in Nitride Semiconductors, Phys. Rev. Lett. 108, 156403 (2012).

[29] S. Kerdsongpanya, B. Alling, and P. Eklund, Effect of point defects on the electronic density of states of $\mathrm{ScN}$ studied by first-principles calculations and implications for thermoelectric properties, Phys. Rev. B 86, 195140 (2012).

[30] C. Stampfl, R. Asahi, and A. J. Freeman, Surface properties of the refractory metal-nitride semiconductor $\mathrm{ScN}$ : Screened-exchange LDA-FLAPW investigations, Phys. Rev. B 65, 161204 (2002).

[31] P. E. Blöchl, Projector augmented-wave method, Phys. Rev. B 50, 17953 (1994).

[32] G. Kresse and D. Joubert, From ultrasoft pseudopotentials to the projector augmented-wave method, Phys. Rev. B 59, 1758 (1999).

[33] G. Kresse and J. Furthmüller, Efficient iterative schemes for ab initio total-energy calculations using a plane-wave basis set, Phys. Rev. B 54, 11169 (1996).

[34] J. Heyd, G. E. Scuseria, and M. Ernzerhof, Hybrid functionals based on a screened coulomb potential, J. Chem. Phys. 118, 8207 (2003).

[35] J. Heyd, G. E. Scuseria, and M. Ernzerhof, Erratum: Hybrid functionals based on a screened coulomb potential, J. Chem. Phys. 124, 219906 (2006).

[36] A. V. Krukau, O. A. Vydrov, A. F. Izmaylov, and G. E. Scuseria, Influence of the exchange screening parameter on the performance of screened hybrid functionals, J. Chem. Phys. 125, 224106 (2006).

[37] P. W. Tasker, The stability of ionic crystal surfaces, J. Phys. C 12, 4977 (1979).

[38] Y. Kumagai, K. T. Butler, A. Walsh, and F. Oba, Theory of ionization potentials of nonmetallic solids, Phys. Rev. B 95, 125309 (2017).

[39] Y. Kumagai, L. A. Burton, A. Walsh, and F. Oba, Electronic Structure and Defect Physics of Tin Sulfides: $\mathrm{SnS}, \mathrm{Sn}_{2} \mathrm{~S}_{3}$, and $\mathrm{SnS}_{2}$, Phys. Rev. Applied 6, 014009 (2016). 
[40] A. Baldereschi, S. Baroni, and R. Resta, Band Offsets in Lattice-Matched Heterojunctions: A Model and FirstPrinciples Calculations for GaAs/AlAs, Phys. Rev. Lett. 61, 734 (1988).

[41] Y. Kumagai and F. Oba, Electrostatics-based finite-size corrections for first-principles point defect calculations, Phys. Rev. B 89, 195205 (2014).

[42] C. Freysoldt, J. Neugebauer, and C. G. Van de Walle, Fully Ab Initio Finite-Size Corrections for Charged-Defect Supercell Calculations, Phys. Rev. Lett. 102, 016402 (2009).

[43] J. P. Perdew, K. Burke, and M. Ernzerhof, Generalized Gradient Approximation Made Simple, Phys. Rev. Lett. 77, 3865 (1996).

[44] S. L. Dudarev, G. A. Botton, S. Y. Savrasov, C. J. Humphreys, and A. P. Sutton, Electron-energy-loss spectra and the structural stability of nickel oxide: An LSDA $+U$ study, Phys. Rev. B 57, 1505 (1998).

[45] H.-P. Komsa, T. T. Rantala, and A. Pasquarello, Finite-size supercell correction schemes for charged defect calculations, Phys. Rev. B 86, 045112 (2012).

[46] Y. Kumagai, M. Choi, Y. Nose, and F. Oba, First-principles study of point defects in chalcopyrite $\mathrm{ZnSnP}_{2}$, Phys. Rev. B 90, 125202 (2014).

[47] H. A. Al-Brithen, A. R. Smith, and D. Gall, Surface and bulk electronic structure of $\mathrm{ScN}(001)$ investigated by scanning tunneling microscopy/spectroscopy and optical absorption spectroscopy, Phys. Rev. B 70, 045303 (2004).

[48] Y. Hinuma, H. Hayashi, Y. Kumagai, I. Tanaka, and F. Oba, Comparison of approximations in density functional theory calculations: Energetics and structure of binary oxides, Phys. Rev. B 96, 094102 (2017).

[49] D. Gall, I. Petrov, P. Desjardins, and J. E. Greene, Microstructural evolution and Poisson ratio of epitaxial $\mathrm{ScN}$ grown on $\mathrm{TiN}(001) / \mathrm{MgO}(001)$ by ultrahigh vacuum reactive magnetron sputter deposition, J. Appl. Phys. 86, 5524 (1999).

[50] B. Saha, J. Acharya, T. D. Sands, and U. V. Waghmare, Electronic structure, phonons, and thermal properties of $\mathrm{ScN}, \mathrm{ZrN}$, and HfN: A first-principles study, J. Appl. Phys. 107, 033715 (2010).

[51] Y. Hinuma, G. Pizzi, Y. Kumagai, F. Oba, and I. Tanaka, Band structure diagram paths based on crystallography, Comput. Mater. Sci. 128, 140 (2017).

[52] S. W. King, R. F. Davis, and R. J. Nemanich, Gas source molecular beam epitaxy of scandium nitride on silicon carbide and gallium nitride surfaces, J. Vac. Sci. Technol. A 32, 061504 (2014).

[53] D. Gall, M. Städele, K. Järrendahl, I. Petrov, P. Desjardins, R. T. Haasch, T.-Y. Lee, and J.E. Greene, Electronic structure of $\mathrm{ScN}$ determined using optical spectroscopy, photoemission, and ab initio calculations, Phys. Rev. B 63, 125119 (2001).

[54] J. M. Gregoire, S. D. Kirby, G. E. Scopelianos, F. H. Lee, and R. B. van Dover, High mobility single crystalline $\mathrm{ScN}$ and single-orientation epitaxial YN on sapphire via magnetron sputtering, J. Appl. Phys. 104, 074913 (2008).

[55] T. Ohgaki, K. Watanabe, Y. Adachi, I. Sakaguchi, S. Hishita, N. Ohashi, and H. Haneda, Electrical properties of scandium nitride epitaxial films grown on (100) magnesium oxide substrates by molecular beam epitaxy, J. Appl. Phys. 114, 093704 (2013).

[56] B. Saha, M. Garbrecht, J. A. Perez-Taborda, M. H. Fawey, Y. R. Koh, A. Shakouri, M. Martin-Gonzalez, L. Hultman, and T. D. Sands, Compensation of native donor doping in $\mathrm{ScN}$ : Carrier concentration control and $p$-type $\mathrm{ScN}$, Appl. Phys. Lett. 110, 252104 (2017).

[57] P. G. Moses, M. Miao, Q. Yan, and C. G. V. de Walle, Hybrid functional investigations of band gaps and band alignments for AlN, GaN, InN, and InGaN, J. Chem. Phys. 134, 084703 (2011).

[58] Y. Hinuma, Y. Kumagai, F. Oba, and I. Tanaka, Categorization of surface polarity from a crystallographic approach, Comput. Mater. Sci. 113, 221 (2016).

[59] A. Jain, S. P. Ong, G. Hautier, W. Chen, W. D. Richards, S. Dacek, S. Cholia, D. Gunter, D. Skinner, G. Ceder, and K. A. Persson, The Materials Project: A materials genome approach to accelerating materials innovation, APL Mater. 1, 011002 (2013).

[60] S. P. Ong, S. Cholia, A. Jain, M. Brafman, D. Gunter, G. Ceder, and K. A. Persson, The materials application programming interface (API): A simple, flexible and efficient API for materials data based on REpresentational State Transfer (REST) principles, Comput. Mater. Sci. 97, 209 (2015).

[61] K. Momma and F. Izumi, vesta3 for three-dimensional visualization of crystal, volumetric and morphology data, J. Appl. Crystallogr. 41, 653 (2008).

[62] C. G. Van de Walle and J. Neugebauer, First-principles calculations for defects and impurities: Applications to III-nitrides, J. Appl. Phys. 95, 3851 (2004).

[63] A. Janotti, J. L. Lyons, and C. G. Van de Walle, Hybrid functional calculations of native point defects in InN, Phys. Status Solidi (a) 209, 65 (2012).

[64] C. Lin and A. A. Demkov, Electron Correlation in Oxygen Vacancy in $\mathrm{SrTiO}_{3}$, Phys. Rev. Lett. 111, 217601 (2013).

[65] M. Choi, F. Oba, Y. Kumagai, and I. Tanaka, Antiferrodistortive-like oxygen-octahedron rotation induced by the oxygen vacancy in cubic $\mathrm{SrTiO}_{3}$, Adv. Mater. 25, 86 (2013).

[66] S. Limpijumnong and C. G. Van de Walle, Diffusivity of native defects in GaN, Phys. Rev. B 69, 035207 (2004).

[67] R. D. Shannon, Revised effective ionic radii and systematic studies of interatomic distances in halides and chalcogenides, Acta Crystallogr. Sect. A 32, 751 (1976).

[68] J. L. Lyons, A. Janotti, and C. G. Van de Walle, Carbon impurities and the yellow luminescence in GaN, Appl. Phys. Lett. 97, 152108 (2010).

[69] N. Hatada, http://www.aqua.mtl.kyoto-u.ac.jp/chesta.html. 